\title{
Free electron laser oscillators with tapered undulators: Inclusion of harmonic generation and pulse propagation
}

\author{
G. Dattoli, ${ }^{1}$ S. Pagnutti, ${ }^{2}$ P. L. Ottaviani, ${ }^{3}$ and V. Asgekar ${ }^{4}$ \\ ${ }^{1}$ Agenzia per le nuove tecnologie e lo sviluppo sostenibile (ENEA), Centro ENEA di Frascati, Frascati Rome, Italy \\ ${ }^{2}$ Agenzia per le nuove tecnologie e lo sviluppo sostenibile (ENEA), Centro ENEA di Bologna, Bologna, Italy \\ ${ }^{3}$ INFN, Sezione di Bologna, Bologna, Italy \\ ${ }^{4}$ Department of Physics, University of Pune, Pune 411007, India \\ (Received 15 November 2011; published 22 March 2012)
}

\begin{abstract}
We review the theory of free-electron laser (FEL) oscillators operating with tapered undulators. We have considered the case of a uniform tapering and introduced a parameter which characterizes the effect of the tapering on the gain and the saturation intensity. We have analyzed the effect of the tapering on the FEL dynamics by including the pulse propagation effects. We further analyze the importance of tapering as a tool to model the optical pulse shapes and to control the higher harmonic intensities.
\end{abstract}

DOI: 10.1103/PhysRevSTAB.15.030708

PACS numbers: 41.60.Cr

\section{INTRODUCTION}

Free-electron laser (FEL) oscillators operating with tapered undulators have been discussed in the past, but the relevant theory and phenomenology require some clarifications because there are some not fully understood aspects, which deserve further consideration. Furthermore, the problems associated with the pulse propagation effects and nonlinear harmonic generation have not been discussed in depth and, as we will report here, they give rise to new and interesting dynamical features which are worth studying carefully.

Originally, the concept of undulator tapering was introduced for FEL amplifiers [1] and its straightforward extension to the oscillator regime has been the source of some surprises, regarding the relevant consequences on the oscillator efficiency.

In the case of the amplifier, the tapering is usually designed in such a way that the undulator field (and/or the undulator period as well) decreases in the forward direction, in order to compensate the effect of the energy losses of the e-beam and ensure an efficient trapping of the electrons in a stable bucket. The increase of the undulator field in the forward direction, namely the reverse tapering, causes reduction in the FEL amplifier efficiency. On the one hand, a mild reverse tapering may be a tool to enhance the efficiency in the case of FEL oscillators [2-4]. The problem of a FEL oscillator with tapered undulators has been the topic of experimental and theoretical investigations, but some interesting aspects have not been explored yet, such as the effect of tapering on the nonlinear harmonic generation. Here we will fill some of these

Published by the American Physical Society under the terms of the Creative Commons Attribution 3.0 License. Further distribution of this work must maintain attribution to the author(s) and the published article's title, journal citation, and DOI. gaps and consider quite a general treatment by including the small and the strong signal regimes. The analysis will, however, be limited to linear tapering and we will not develop strategies for an optimal tapering, which is not a well-defined concept within the framework of the present investigation.

This paper combines analytical and numerical results and is also devoted to the derivation of practical formulas, which include the effect of the tapering on the gain and the saturation intensity. The paper consists of two parts.

In the first we will establish practical formulas concerning the FEL operation with a linear tapering (namely, with undulators exhibiting an on axis field amplitude depending linearly on the longitudinal coordinate). In particular, we will derive the expressions for the gain and the efficiency factor as a function of tapering depth, the gain saturation formula, and the saturation intensity.

In the second part we will discuss the pulse propagation effects and the interplay between slippage, short pulses, and tapering. We will investigate how the tapering combines with slippage and lethargy effects, to give rise to a new and interesting phenomenology.

\section{SMALL SIGNAL GAIN INCLUDING TAPERING}

In this section we will discuss different levels of approximation concerning the tapered FEL phenomenology. We will indeed consider the small signal low gain effects and we will essentially recover the results of Ref. [2]. We will then include the high gain corrections and the consequences of the pulse propagation effects.

Following Ref. [2], we write

$$
\begin{aligned}
G_{T}\left(\nu, \mu_{T}\right) & =-2 \pi g_{0} \operatorname{Im}\left[g\left(\nu, \mu_{T}\right)\right], \\
g\left(\nu, \mu_{T}\right) & =\int_{0}^{1} d \tau \int_{0}^{\tau} d \xi \xi e^{-i \nu \xi+i\left(\pi \mu_{T} / 2\right) \xi(2 \tau-\xi)},
\end{aligned}
$$

where 

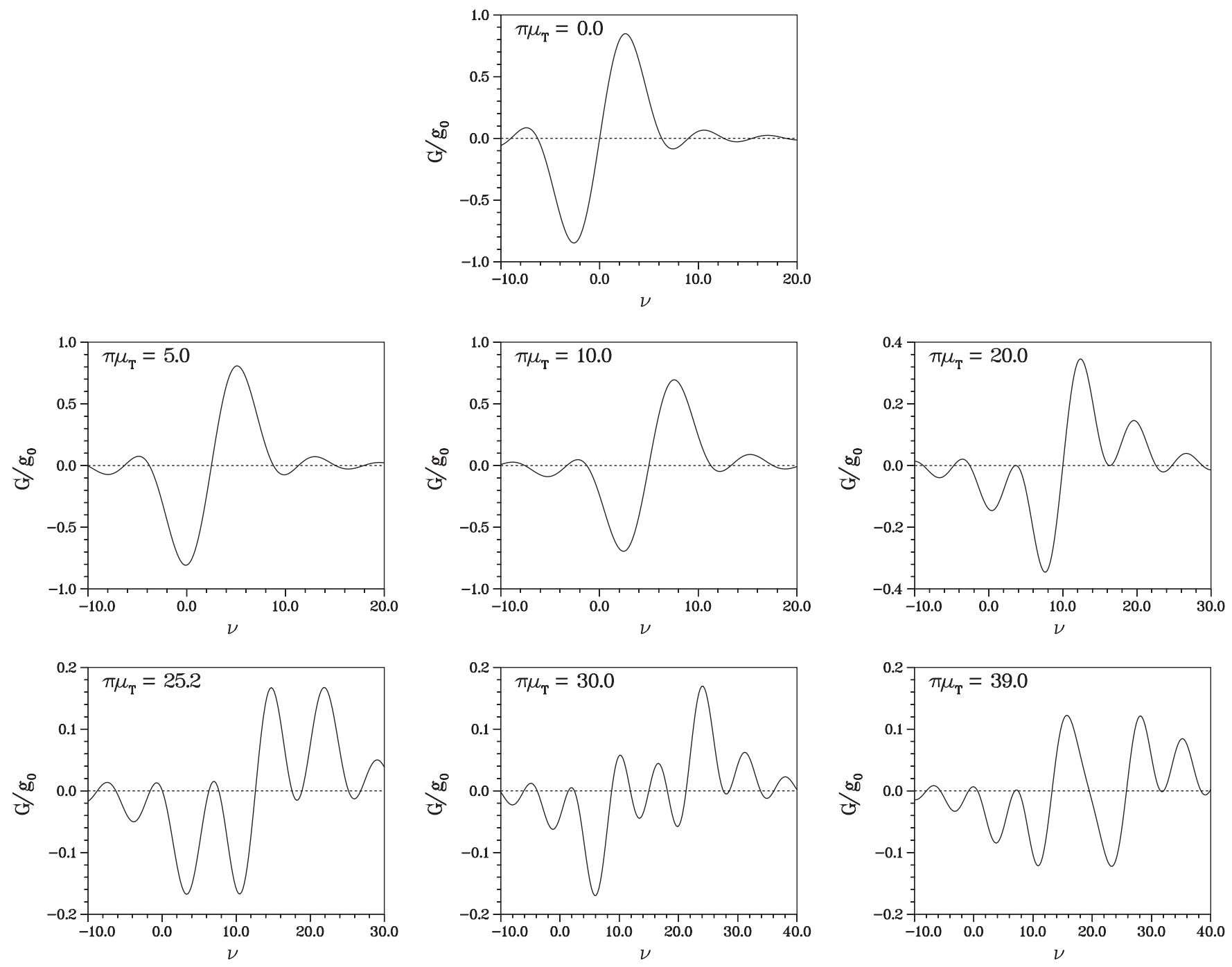

FIG. 1. Gain curves vs frequency detuning for different $\mu_{T}$.

$$
\mu_{T}=2 N \frac{\Delta B}{B} \frac{K_{0}^{2}}{1+\frac{K_{0}^{2}}{2}}, \quad \Delta B=B\left(L_{u}\right)-B(0)
$$

$N$ is the number of undulator periods, $L_{u}=N \lambda_{u}$ is the undulator length, $\mu_{T}$ is the tapering parameter, $\frac{\Delta B}{B}$ is the field variation along the undulator with a uniform tapering, and $K_{0}$ is the undulator parameter at the entrance of the undulator. ${ }^{1}$

The physical meaning of the $\mu_{T}$ parameter should be understood as follows. The phase matching condition, after any undulator period advance, requires that the electron longitudinal velocity, the undulator period, and the FEL wavelength be related by $\left(1-\beta_{z}\right) \lambda_{u}=n \lambda$ to ensure constructive interference. In the case of tapered devices the

\footnotetext{
${ }^{1}$ Equation (2) implicitly assumes that the undulator field only undergoes a variation along the longitudinal direction, more in general the undulator period can be tapered too, in this case $\frac{\Delta B}{B}$ in Eq. (2) should be replaced by $\frac{\Delta K}{K}$.
}

longitudinal component of the velocity depends on the position inside the undulator, namely $\beta_{z} \simeq 1-\frac{1}{2 \gamma^{2}} \times$ $\left(1+\frac{K^{2}(z)}{2}\right)$. Therefore, for the linear undulator tapering, the phase matching condition cannot be satisfied throughout the undulator length. This phase mismatch due to undulator tapering gives rise to frequency shift. By assuming linear tapering we can set $K(z)=K_{0}(1+\alpha z)$ where $\alpha=\frac{\Delta B}{B} \ll 1$, which yields a frequency detuning shift given by $\delta \nu \simeq 2 \pi \mu_{T}$. This factor plays a role similar to the inhomogeneous broadening effects and indeed it determines a shift of the maximum gain position and a gain reduction.

As already remarked, the condition of positive $\alpha$ is referred to in literature as "inverse tapering" to denote the fact that the strength of the field increases with increasing $z$. The gain vs the frequency detuning $\nu$ is shown in Fig. 1 for different values of the tapering parameter $\mu_{T}$.

It is evident that when $\mu_{T}$ is small, the gain curve resembles, apart from a shift in the detuning parameter, 


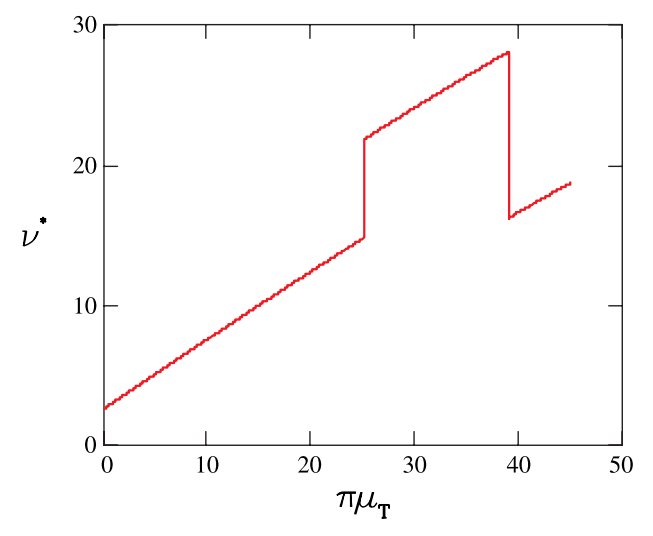

FIG. 2. $\nu^{*}$ vs $\pi \mu$; the plot includes the region above the instability threshold.

the usual low gain curve. When the tapering parameter increases, the maximum gain shifts smoothly towards larger frequency detuning. Further increase corresponds, however, to an abrupt variation of the maximum gain position. The threshold for the occurrence of this effect is $\left|\pi \mu_{T}\right| \simeq 25.2$.

The value of the detuning, corresponding to the maximum gain, including the corrections due to the tapering, is given by the following simple relation:

$$
\begin{aligned}
\nu^{*}\left(\mu_{T}\right) & \simeq \nu^{*}(0)\left[1+0.1844 \pi \mu_{T}\right], \\
\left|\pi \mu_{T}\right| & <25.2, \quad g_{0} \leq 0.3
\end{aligned}
$$

and is shown in Fig. 2.

For values of the tapering parameter larger than 25.2, the curve exhibits a kind of discontinuity due to a longitudinal mode flipping. The consequences of this behavior will be commented on later in the paper. The dependence of the maximum gain on the tapering parameter is shown in Fig. 3, where the discontinuity in the detuning parameter is clearly evident.

The maximum gain function for $\left|\pi \mu_{T}\right| \leq 25.2$ is reproduced by the equation

$$
\begin{aligned}
G_{M}\left(\mu_{T}\right) & \simeq G_{M}\left(g_{0}\right) P\left(\mu_{T}\right), \quad G_{M}\left(g_{0}\right) \simeq 0.848 g_{0} \\
P\left(\mu_{T}\right) & =1+0.275\left[1-\frac{2}{1+e^{0.301\left(\pi \mu_{T}\right)}}\right]-0.0428 \pi \mu_{T} .
\end{aligned}
$$

For the larger values of the tapering parameter $\left(25.3 \leq \pi\left|\mu_{T}\right| \leq 39\right),{ }^{2}$ the maximum gain is almost constant and exhibits the following parabolic form:

\footnotetext{
${ }^{2} \mathrm{We}$ will not consider values of the tapering above the first threshold because the gain becomes too small and the effects of the tapering have no practical importance. It was however unexpected to find the occurrence of a further peak shift and for this reason we have quoted such extreme values.
}

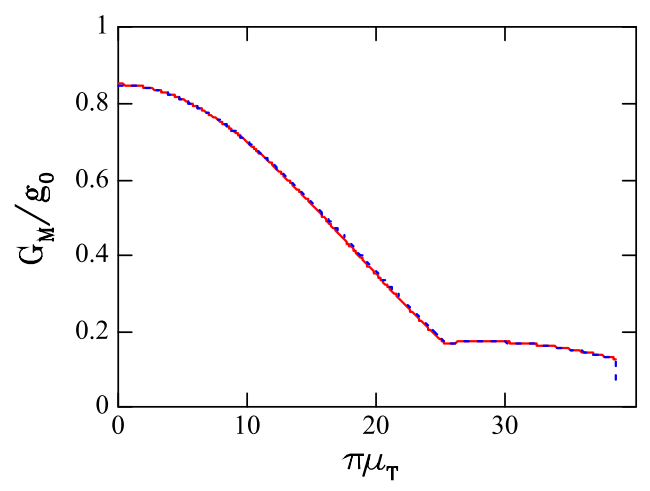

FIG. 3. $\frac{G_{M}}{g_{0}}$ vs $\pi \mu_{T}$; the dotted line is the fitting curve.

$$
\begin{aligned}
G_{M}\left(\mu_{T}\right) \simeq & 0.168 \cdot g_{0} Q\left(\mu_{T}\right) \\
Q\left(\mu_{T}\right)= & 1+13.69 \times 10^{-3}\left(\pi \mu_{T}-25.3\right) \\
& -25 \times 10^{-4}\left(\pi \mu_{T}-25.3\right)^{2} .
\end{aligned}
$$

The results obtained so far confirm those of Ref. [2] and show that the role of tapering is fairly more complicated than usually believed. The gain exhibits indeed a rich structure, which, as we will see in the following, is responsible for an unexpected and interesting dynamical behavior of FEL oscillators operating with nonconstant parameter undulators.

The considerations we have developed so far do not include high gain effects, which can be accounted for using the integral equation:

$$
\partial_{\tau} a=i \pi g_{0} \int_{0}^{\tau} d \xi \xi e^{-i \nu \xi+\left(i \pi \mu_{T} / 2\right) \xi(2 \tau-\xi)} a(\tau-\xi) .
$$

It is well known, from the usual FEL gain theory, that, when the small signal gain coefficient increases, the gain curve loses its antisymmetric shape. An example of this is reported in Fig. 4 where we have shown the gain of a FEL operating with a large small signal gain $g_{0}=2$ and a modest value of the tapering parameter. This means that, for increasing $g_{0}$, nonlinear contributions in the gain coefficient play an increasingly important role. It has, however, been shown that these high gain contributions tend to disappear when inhomogeneous broadening due to energy spread and/or emittance are active [5,6]. This effect occurs with the increase of the tapering parameter too, as shown in Fig. 4.

We have checked the validity of the previous gain curves by evaluating the gain dependence vs detuning dependence by means of the simulation code PROMETEO [5]. The code has been run with the parameters reported in Table I. The comparison, discussed below, confirms the correctness of the previous analysis.

For large values of the small signal gain coefficient, the previous formulas for the value of the detuning $\nu^{*}$ corresponding to the maximum gain and for the maxim gain $G_{M}$ need slight corrections in $g_{0}$, reported below: 

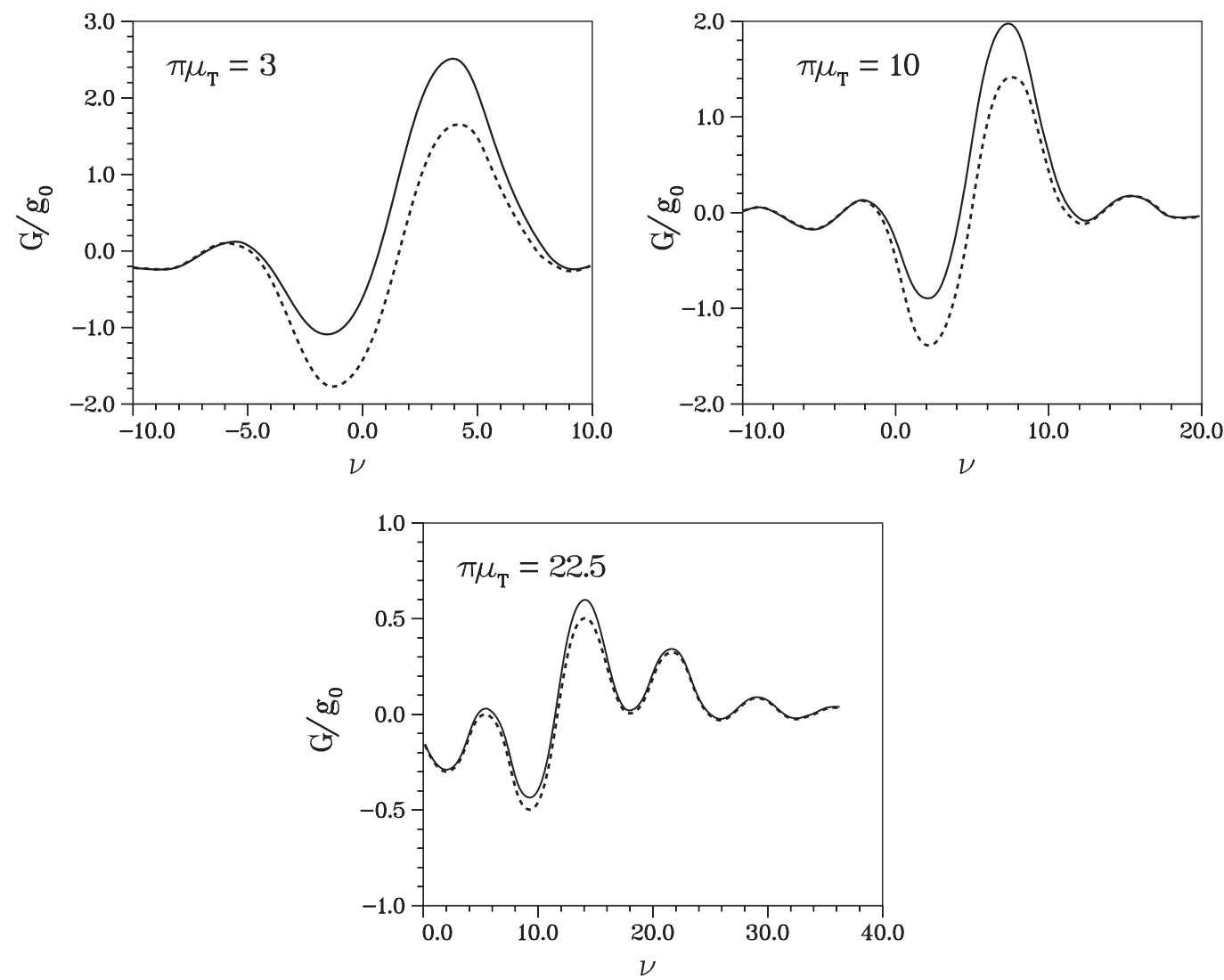

FIG. 4. Gain vs detuning for $g_{0}=2$ and different values of $\pi \mu_{T}$; continuous line with high gain corrections, dotted without high gain corrections.

$$
\begin{aligned}
\nu^{*}\left(g_{0}, \mu_{T}\right) & \simeq \nu^{*}\left(g_{0}, 0\right)\left[1+0.1832\left(1+0.0631 g_{0}\right) \pi \mu_{T}\right], \\
G_{M}\left(g_{0}, \mu_{T}\right) \simeq & G_{M}\left(g_{0}\right) P\left(g_{0}, \mu_{T}\right), \\
G_{M}\left(g_{0}\right) \simeq & 0.848 g_{0}+0.19 g_{0}^{2} \\
P\left(g_{0}, \mu_{T}\right)= & 1+0.275\left[1-\frac{2}{1+e^{0.301\left(\pi \mu_{T}\right)}}\right] \\
& -0.0428\left(1+0.042 g_{0}\right) \pi \mu_{T}, \quad g_{0}<2 .
\end{aligned}
$$

In Fig. 5 we have shown the behavior of gain vs $\pi \mu_{T}$ for different values of $g_{0}$.

Reversing the sign of the tapering does not create significant difference (at least in the small signal regime), as shown in Fig. 6, where the gain curve with opposite

TABLE I. Simulation parameters.

\begin{tabular}{lc}
\hline \hline$E[\mathrm{MeV}]$ & 155.3 \\
\hline$\lambda_{u}[\mathrm{~cm}]$ & 2.8 \\
$K_{0}$ & 2.13 \\
$N$ & 50 \\
Relative energy spread & $10^{-4}$ \\
e-bunch length $[\mu \mathrm{m}]$ & $10^{2}$ \\
Cavity length $[\mathrm{m}]$ & 2 \\
\hline \hline
\end{tabular}

tapering values has the same shape, but its maximum is located at $\nu^{*}(0)\left[1-0.1844 \cdot\left|\pi \mu_{T}\right|\right]$.

Before concluding this section it is worth making some comments on the agreement between the gain curves

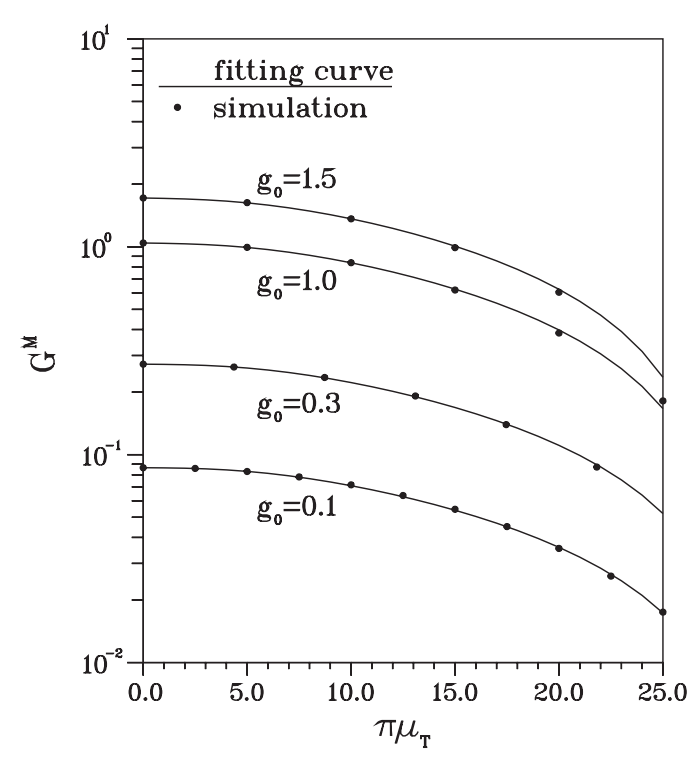

FIG. 5. Maximum gain vs $\pi \mu_{T}$ for different values of $g_{0}$; the dots are the result of the simulation, the continuous curve is the fitting curve [Eq. (7)]. 


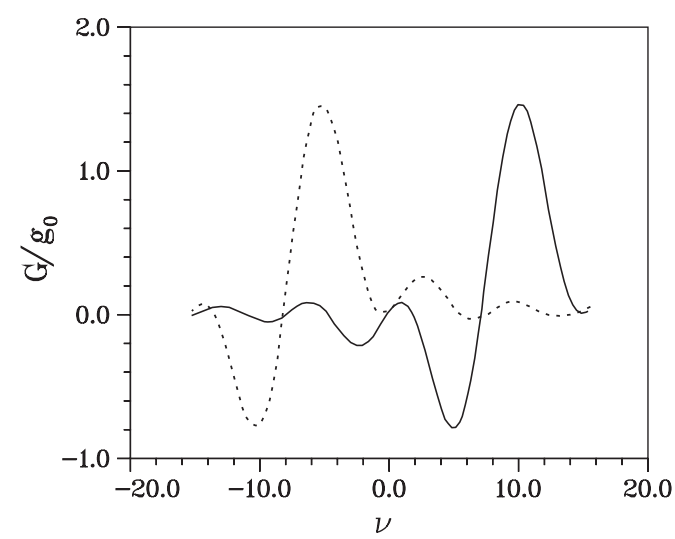

FIG. 6. Gain vs detuning for $g_{0}=2$; continuous line $\pi \mu_{T}=$ 15, dotted line $\pi \mu_{T}=-15$.

obtained with the numerical code and the integral equation. An example is shown in Fig. 7. The disagreement between analytical and numerical solution is due to the fact that the analytical procedure does not provide any corrections associated with the variations of the $K$ parameter in the small signal gain coefficient. The relative error associated with the Bessel factor term in the gain coefficient is proportional to $\left(\frac{\Delta K}{2 K}\right)^{2}$ and becomes more significant with the increasing of the tapering parameter.

\section{THE SATURATION INTENSITY}

In the previous section we have dealt with the small signal regime. Here we will introduce the saturation effects and discuss how other quantities of crucial importance for the FEL performance, like the saturation intensity, are affected by the tapering.

We would like to mention here that, by saturation intensity, we mean the value of the laser intensity for which the FEL gain becomes half of its small signal gain value.

The gain saturation mechanism consists essentially of two parts; one which is a kind of frequency shift towards the negative part of the gain and the second is an effect of

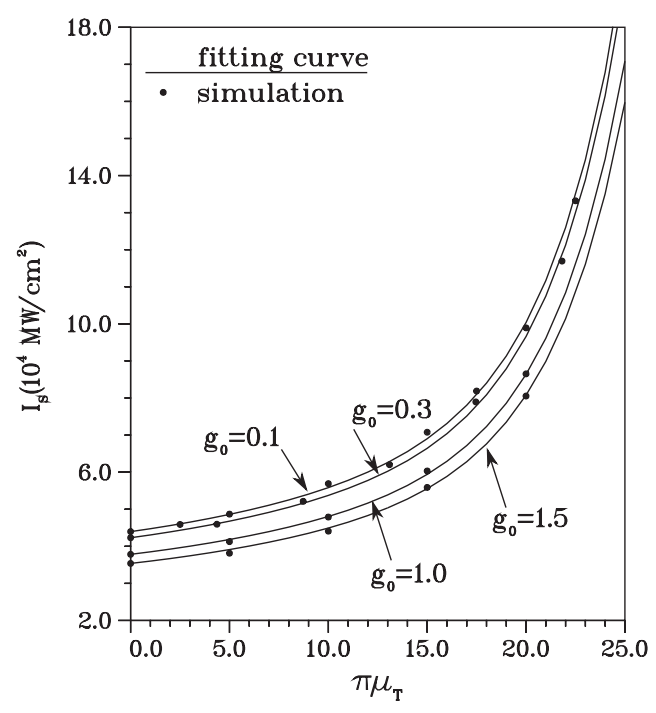

FIG. 8. Saturation intensity vs tapering parameter for different $g_{0}$; the dots are the result of the simulation, the continuous curve is the fitting curve [Eq. (9)].

the inhomogeneous broadening associated with the induced energy spread.

We introduce the FEL Hamiltonian, which can be quite useful to understand the previously mentioned points. For a tapered undulator FEL the Hamiltonian can be written, according to Ref. [7], as

$$
H=\frac{1}{2} \nu^{2}+\pi \mu_{T} \zeta-|a| \sin (\zeta+\varphi)
$$

where the effect of tapering on the FEL dynamics is like that of an accelerating electric field. The above Hamiltonian is a rigorous formulation of this effect.

The fact that the gain is shifted towards larger $\nu$ values implies that it requires larger kinetic energy to overcome the trapping potential. For this reason the saturation intensity should be larger than the corresponding value for the nontapered case.

In Fig. 8 we have reported the saturation intensities vs tapering parameter for different values of $g_{0}$ and the corresponding behavior is reproduced by

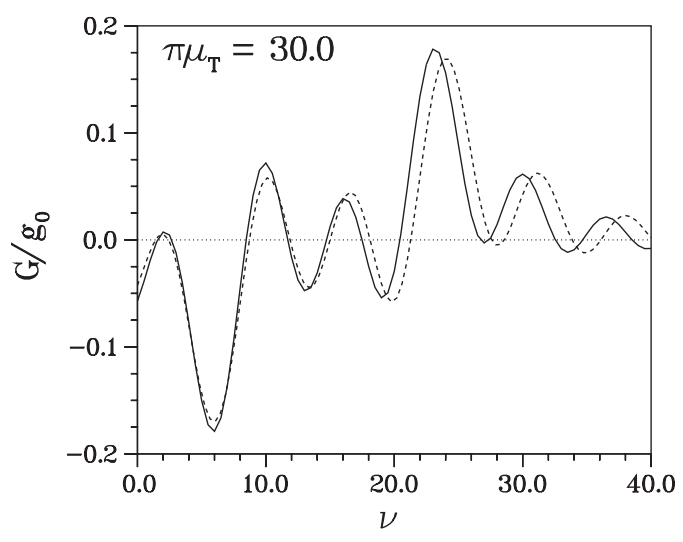

FIG. 7. Gain vs detuning for $g_{0}=0.1$ and different $\pi \mu_{T}$; dotted line: analytical formula; continuous line: simulation. 


$$
\begin{gathered}
\frac{I_{s}\left(g_{0}, \mu_{T}\right)}{I_{s}\left(g_{0}, 0\right)}=1+0.017 \pi \mu_{T}+0.162\left(e^{4.8 \times 10^{-3}\left(\pi \mu_{T}\right)^{2}}-1\right), \\
g_{0}<2 .
\end{gathered}
$$

The saturation intensity $I_{s}\left(g_{0}, 0\right)$ contains the high gain corrections [5]. We stress that Eq. (9) is valid only for reverse tapering. In the case of direct tapering (i.e. for negative values of $\pi \mu_{T}$ ), the situation is different and the associated saturation mechanisms will be discussed elsewhere.

We can combine gain formula and saturation intensity to get the round-trip evolution of the intracavity field $I\left(r_{t}\right)$. The result can be expressed in terms of the discrete logistic equation as [8]

$$
\begin{aligned}
I\left(r_{t}\right) & =I_{0} \frac{\left[(1-\eta)\left(G_{M}\left(g_{0}, \mu_{T}\right)+1\right)\right]^{r_{t}}}{1+\frac{I_{0}}{I_{e}\left(g_{0}, \mu_{T}, \eta\right)}\left\{\left[(1-\eta)\left(G_{M}\left(g_{0}, \mu_{T}\right)+1\right)\right]^{r_{t}}-1\right\}}, \\
I_{e}\left(g_{0}, \mu_{T}, \eta\right) & =(\sqrt{2}+1) h\left(\mu_{T}, \eta / g_{0}\right)\left[\sqrt{\frac{1-\eta}{\eta} G_{M}\left(g_{0}, \mu_{T}\right)}-1\right] I_{s}\left(g_{0}, \mu_{T}\right), \\
h\left(\mu_{T}, \eta / g_{0}\right) & =1+a\left(\mu_{T}\right) e^{-b\left(\mu_{T}\right)\left(n / g_{0}\right)}, \quad \pi \mu_{T}<20, \quad \frac{\eta}{g_{0}}>0.04 \\
a\left(\mu_{T}\right) & =0.124+0.063 e^{0.262 \pi \mu_{T}} \quad b\left(\mu_{T}\right)=9.13-0.34 \pi \mu_{T}+0.068\left(\pi \mu_{T}\right)^{2},
\end{aligned}
$$

where $I_{0}, I_{e}\left(g_{0}, \mu_{T}, \eta\right)$ denote the input seed and the equilibrium intracavity intensities, respectively. The function $h\left(\mu_{T}, \eta / g_{0}\right)$ is an ad hoc introduced correction, which accounts for the high intracavity equilibrium power, occurring at low cavity losses. The comparison between fitting formula and numerical results for $I_{e}$ is reported in Fig. 9 .

The cavity losses can be divided in passive $\eta_{P}$ and active $\eta_{A}$ losses in such a way that

$$
\eta=\eta_{A}+\eta_{P}=\eta_{A}(1+r), \quad r=\frac{\eta_{p}}{\eta_{A}}
$$

and

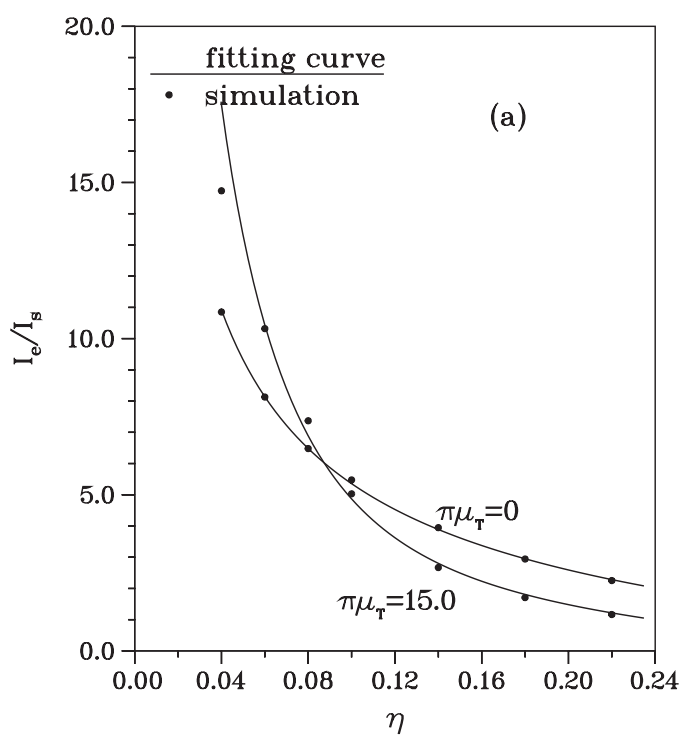

$$
\begin{aligned}
I_{\mathrm{out}}\left(\mu_{T}, \eta_{A}, r\right) & =\eta_{A} I_{e}\left(\mu_{T}, \eta\right)\left(1+G_{e}\right) \\
& =\frac{\eta_{A}}{1-\eta} I_{e}\left(\mu_{T}, \eta\right),
\end{aligned}
$$

where $G_{e}=\frac{\eta}{1-\eta}$ is the equilibrium gain.

The output power is therefore just given by the product of the active losses times the intracavity power density. In Figs. 10 and 11 we have reported the dimensionless output power vs the active losses for different values of the tapering parameter. Figure 10 is the result of a semianalytical computation based on the previous formulas while Fig. 11 shows the simulation results obtained with PROMETEO.

The conclusion we may draw from Figs. 10 and 11 is that the optimum value of the cavity losses depends on the

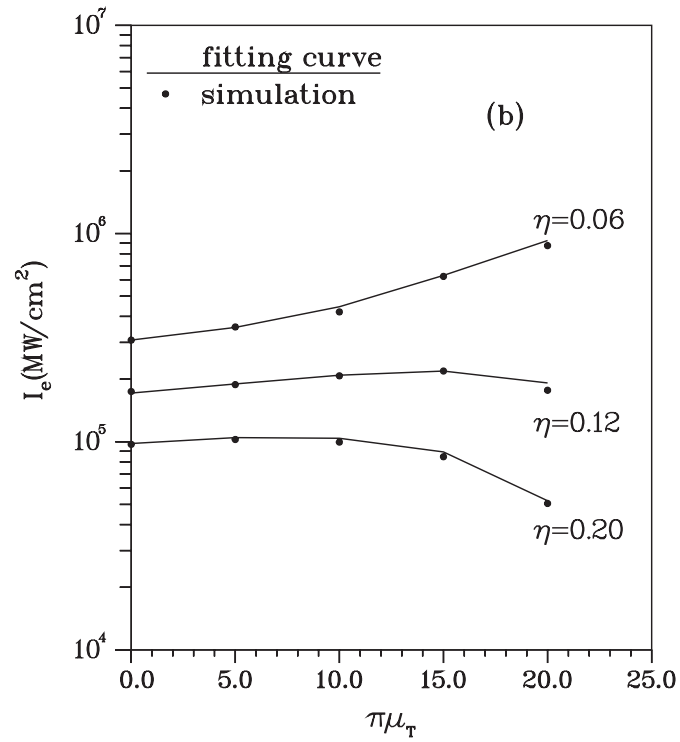

FIG. 9. Intracavity equilibrium power; comparison between numerical results (dots) and fitting formula (continuous line) for $g_{0}=1$ : (a) vs cavity losses, (b) vs tapering parameter. 


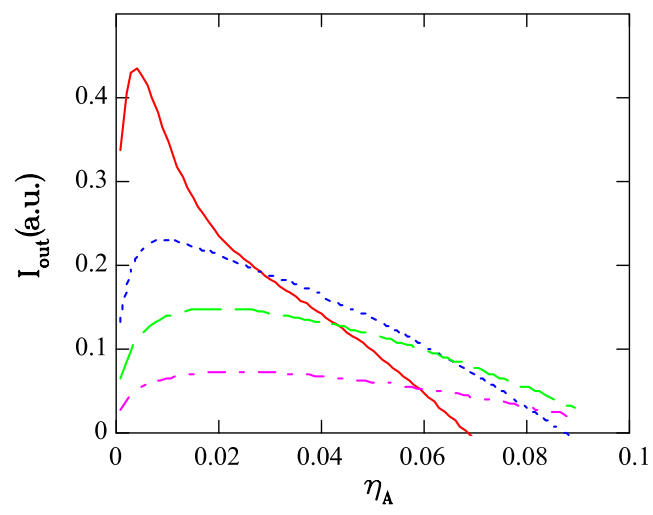

FIG. 10. Dimensionless output power density vs $\eta_{A}, r=0.4$, $g_{0}=0.2 ; \pi \mu_{T}=15$, continuous line; $\pi \mu_{T}=10$, dotted line; $\pi \mu_{T}=5$, dashed line; $\pi \mu_{T}=0$, dash-dotted line.

value of the tapering; this is not surprising since the tapering affects the peak gain. Even if present results are fairly accurate they are the consequence of a one-dimensional analysis. Inclusion of the transverse optical mode structure could induce some changes which do not however hamper the conclusion of the paper.

Let us now discuss whether a tapered undulator determines an effective enhancement of the FEL efficiency, in the case of the oscillator configuration. To this aim we remind that the efficiency is defined as the ratio between the FEL output power density and electron beam power density $I_{E}$, namely,

$E\left(\mu_{T}\right)=\frac{I_{\mathrm{out}}\left(\mu_{T}, \eta_{A}, r\right)}{I_{E}} \quad E(0)=\frac{I_{\mathrm{out}}\left(0, \eta_{A}, r\right)}{I_{E}}$.

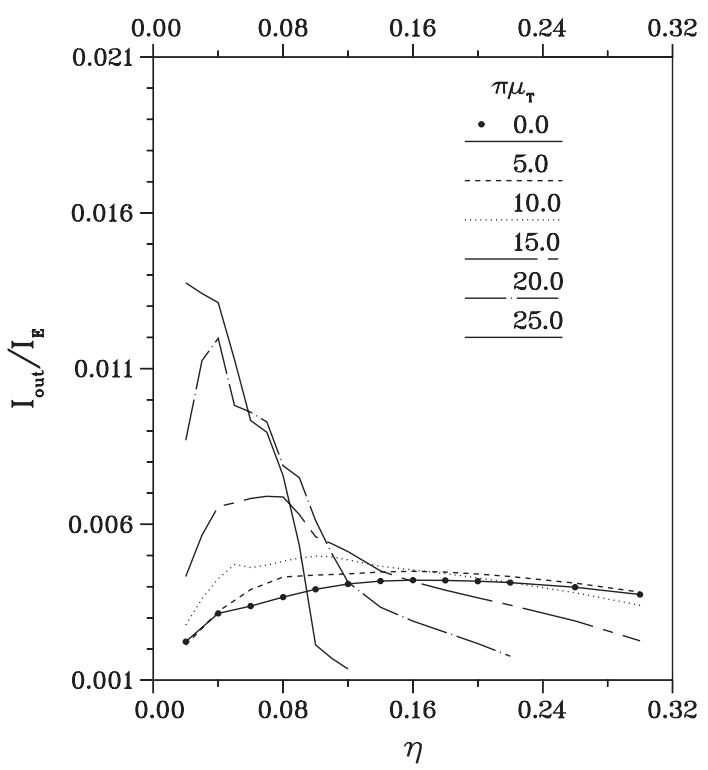

FIG. 11. Ratio of output power to electron power vs losses for different $\pi \mu_{T} ; r=0.4, g_{0}=1$.

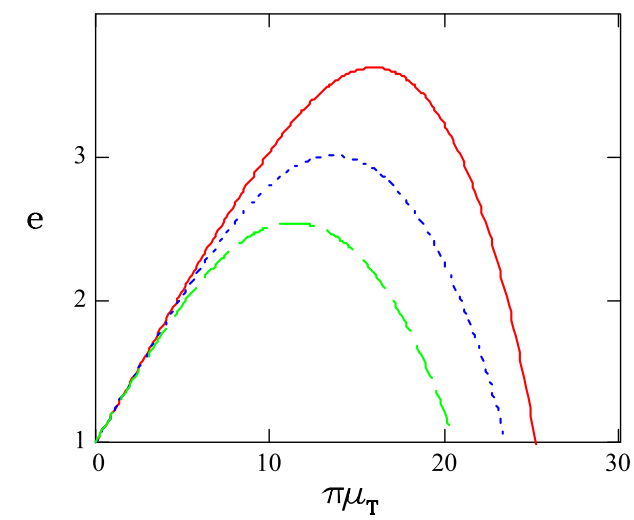

FIG. 12. Tapered FEL efficiency enhancing factor vs $\pi \mu_{T}$ for different values of the active losses, $g_{0}=0.3, r=0.2, \eta_{A}=3 \%$ continuous line; $\eta_{A}=4 \%$ dotted line; $\eta_{A}=6 \%$ dashed line.

The efficiency enhancing factor is

$$
e\left(\mu_{T}, \eta_{A}, r\right)=\frac{I_{\mathrm{out}}\left(\mu_{T}, \eta_{A}, r\right)}{I_{\mathrm{out}}\left(0, \eta_{A}, r\right)}=\frac{E\left(\mu_{T}\right)}{E(0)}
$$

An effective increase of the efficiency due to the tapering occurs therefore whenever $e\left(\mu_{T}, \eta_{A}, r\right)>1$.

In Fig. 12 we have reported the efficiency enhancing factor vs the tapering parameter; it is evident an effective increase $^{3}$ for values of $\pi \mu_{T}$ above 10 .

This assessment is consistent with the recent experimental results of Ref. [4].

Such a comparison cannot be considered conclusive because the analysis developed so far is one-dimensional and not fully appropriate for the analysis of the results of the ELBE FIR tapered FEL oscillator, operating with a waveguide and a hybrid cavity with an output toroidal holed mirror.

\section{PULSE PROPAGATION EFFECTS}

This concluding section is not dedicated to the efficiency problem but to the phenomenology emerging from the inclusion of pulse propagation effects in a tapered FEL oscillator [9]. It is particularly interesting because, as we will see in the following, new interesting dynamical features emerge which make the uniform tapering an additional tool to control the laser beam quality.

The equation yielding the small signal evolution of the FEL field with the inclusion of tapering and short pulses can be written as

\footnotetext{
${ }^{3}$ Note that Fig. 12 shows the increase of efficiency with respect to the value with zero tapering parameter. It does therefore not imply that the optimum of the operation is at $3 \%$ cavity losses, but only that for this value of the active losses the tapering is more efficient.
} 

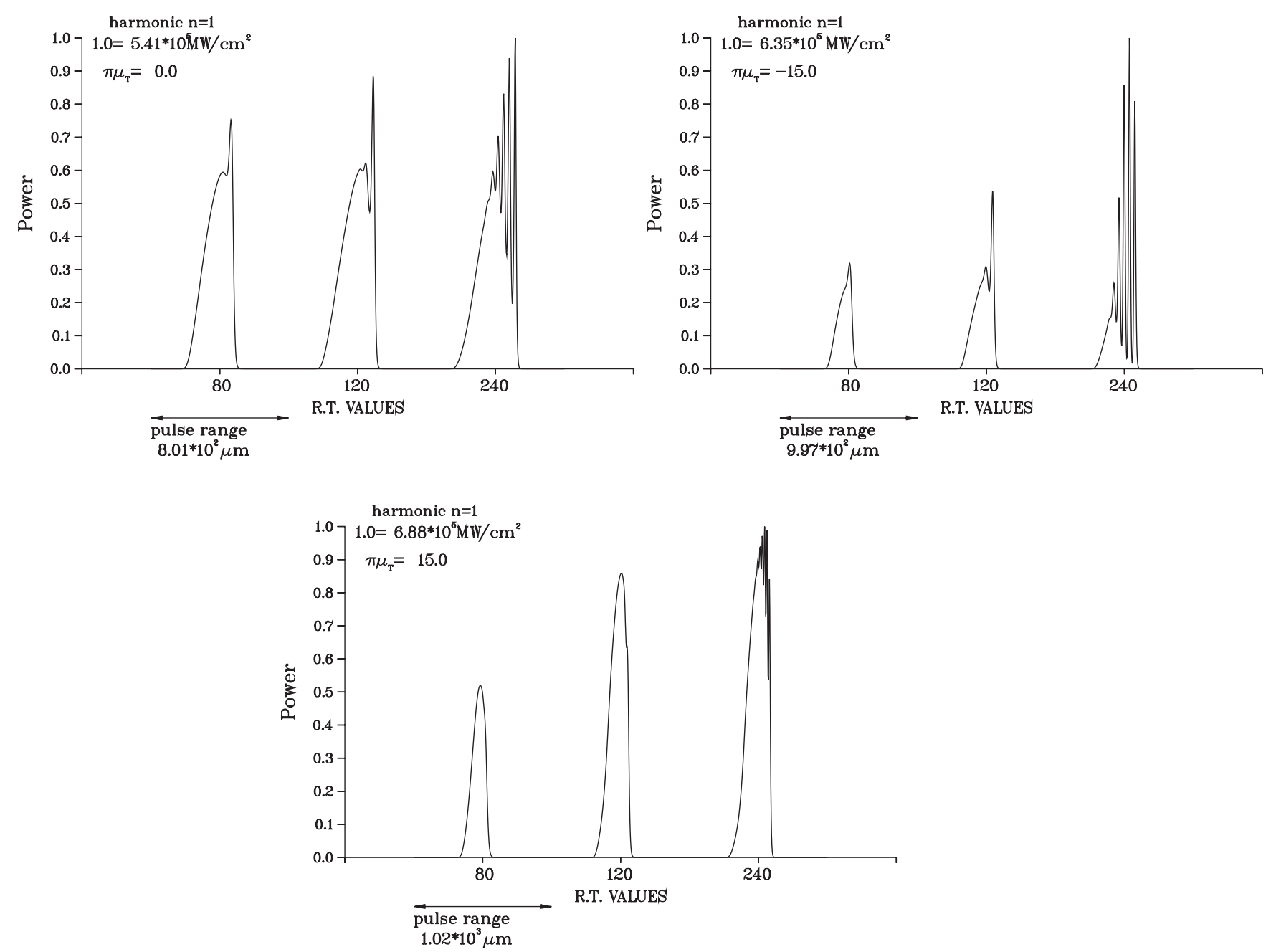

FIG. 13. Optical pulses vs round-trip for different values of $\pi \mu_{T} ; g_{0}=1, \eta=0.06$ and zero cavity mismatch. Pulse range shows Gaussian e-beam range; $\mathrm{x}$-axis tick marks indicate the position of the e-beam center.

$$
\begin{aligned}
\partial_{\tau} a= & i \pi g_{0}(z+\Delta \cdot \tau) \\
& \times \int_{0}^{\tau} d \xi \xi e^{-i \nu \xi+\left(i \pi \mu_{T} / 2\right) \xi(2 \tau-\xi)} a(z+\Delta \cdot \xi, \tau-\xi) \\
\Delta= & N \lambda \equiv \text { slippage length, } \quad g_{0}(z)=g_{0} f(z) \\
f(z) \equiv & \text { electron packet shape. }
\end{aligned}
$$

The slippage length is due to the different velocities between electrons and radiation, which causes a slipping of the optical bunch over the electrons during the interaction inside the undulator. The combination of tapering and slippage gives rise to a new phenomenology.

Albeit we will discuss these aspects of the problem in a dedicated paper, here we will present a few interesting results emerging from the numerical treatment of the problem. In Fig. 13 we have reported the evolution vs the roundtrip of the optical pulses, for the case with zero, negative, and positive values of the tapering parameter and the cavity set at zero mismatch. The sequence shows that the effect of the tapering is an obvious reduction of the small signal gain

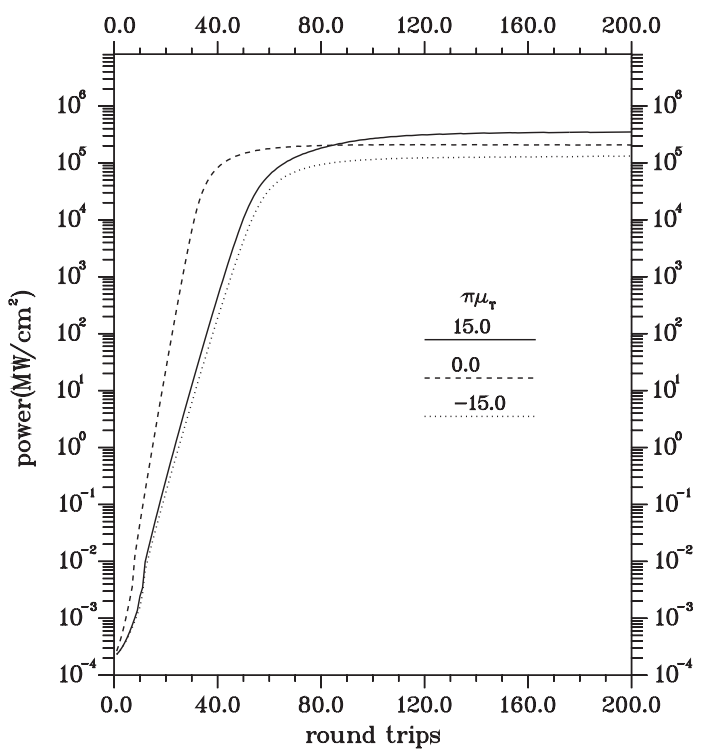

FIG. 14. Intracavity power evolution vs round-trip number for different $\pi \mu_{T}$, same parameters as in Fig. 13. 

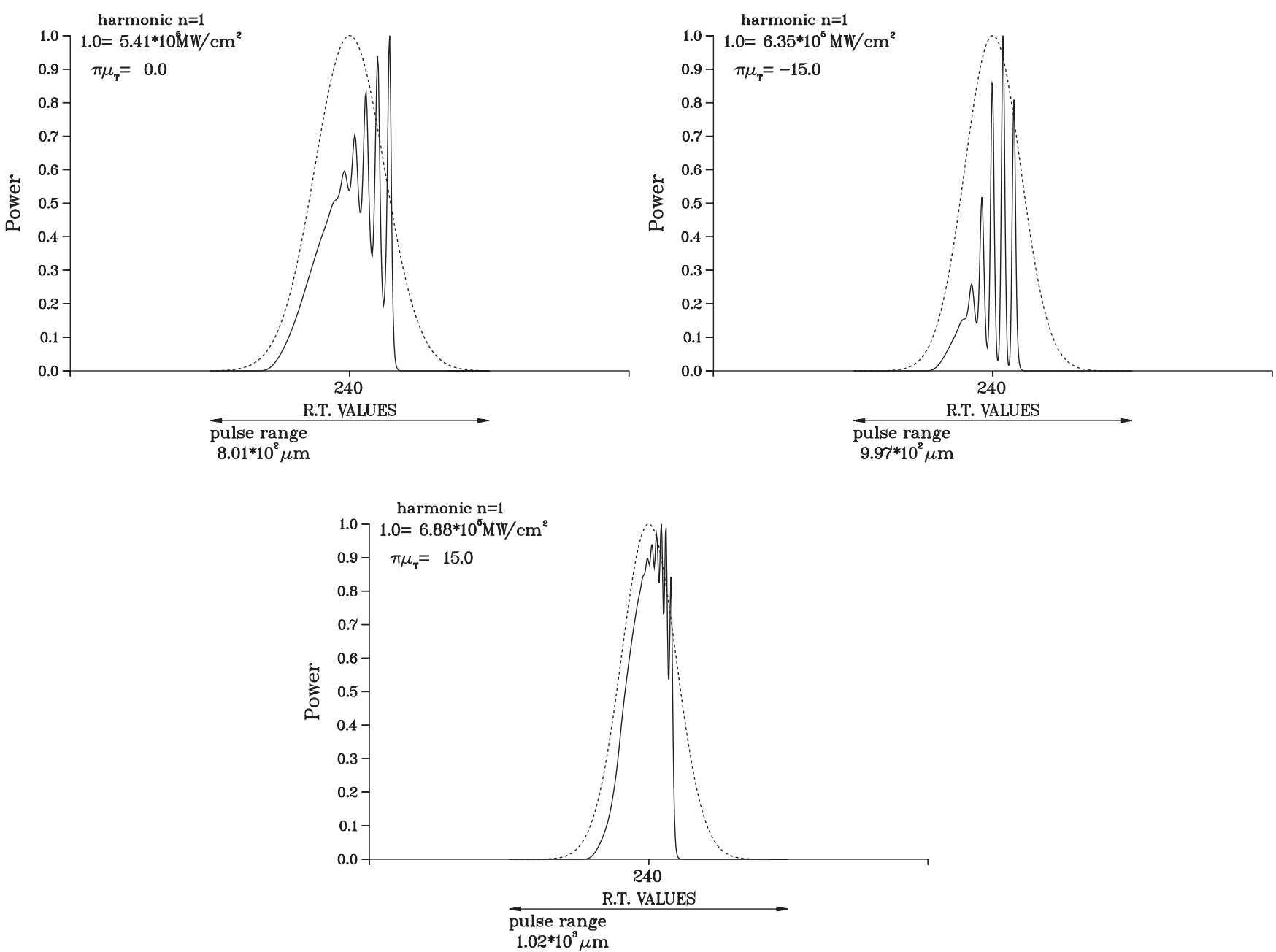

FIG. 15. Comblike structure in FEL oscillators in deep saturation (round-trip 240) at zero cavity mismatch, $g_{0}=1, \eta=0.06$, for different values of $\pi \mu_{T}$; dotted curve represents the electron longitudinal distribution.
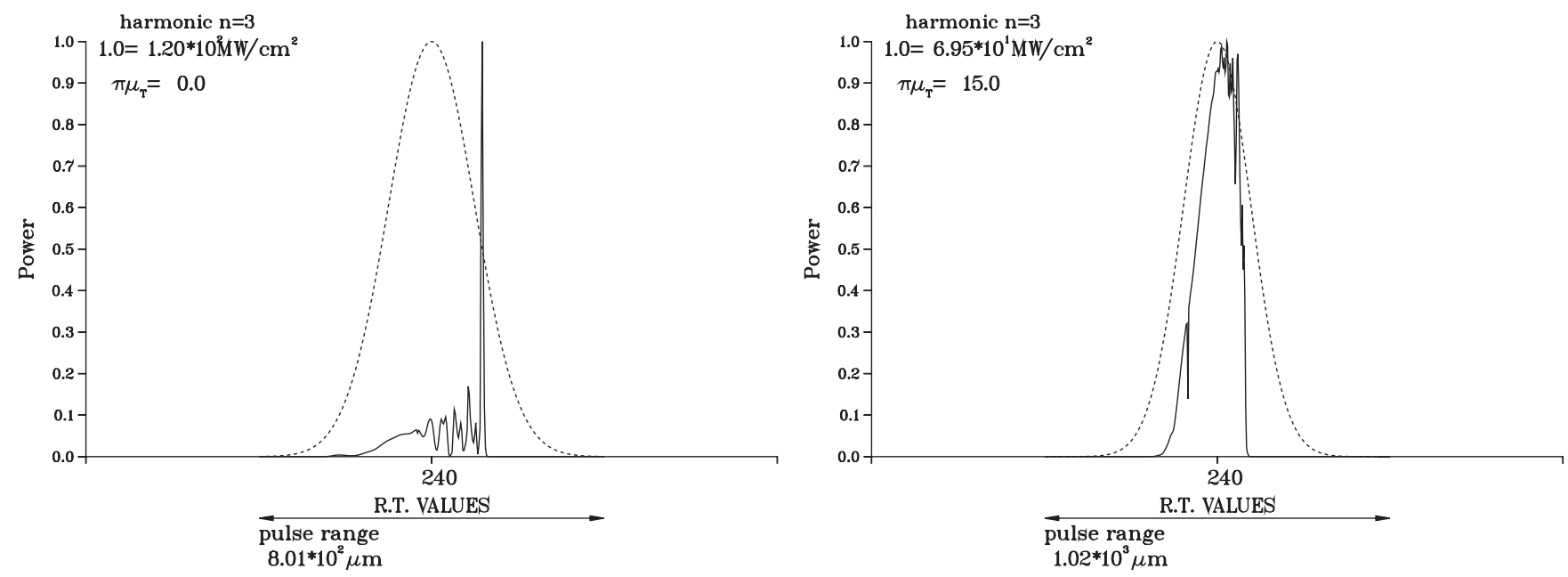

FIG. 16. Third harmonic in deep saturation, same parameters as in Fig. 15, for two different values of $\pi \mu_{T}$. 


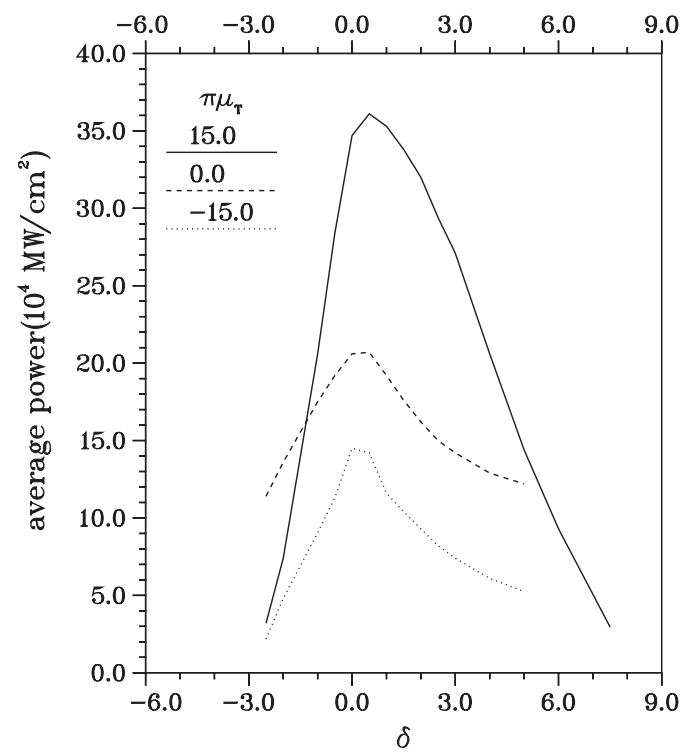

FIG. 17. Equilibrium intracavity power intensity vs $\delta, g_{0}=1$, $\eta=0.06$. The average power is the average on laser packet distribution.

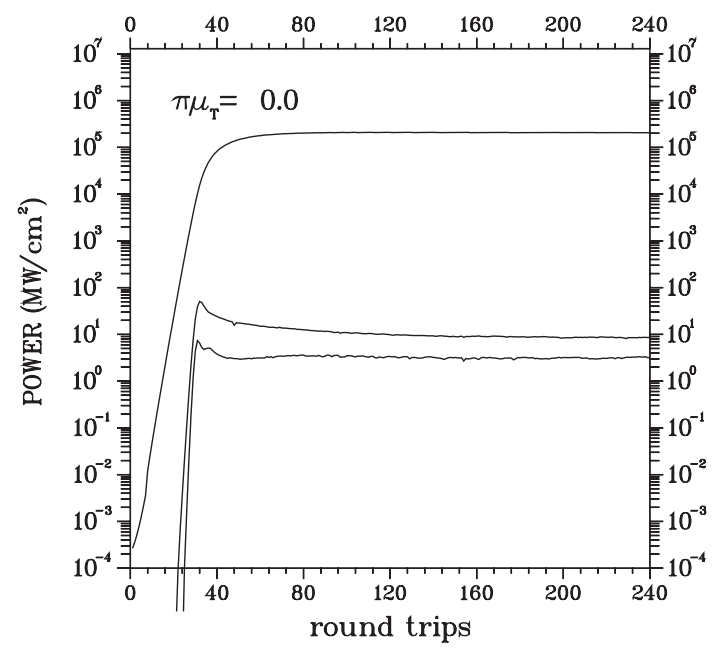

which determines an increase of the time necessary to reach the saturation (see also Fig. 14) and, for nonzero values of the tapering parameter, a kind of compensation of the slippage effect.

The radiation packets tend to be more confined within the electron packet; this effect is more evident for reverse tapering.

In Fig. 15 we have detailed the pulse shapes of the previous snap shots in the region of deep saturation. It is interesting to note that a kind of comblike structure occurs well beyond saturation.

The physical origin of the peaks is essentially due to a strong mode-locking mechanism occurring at the scale of the cooperation length. The effect is a genuine combination between mode locking, slippage effect, and superradiance. The longitudinal mode locking occurs because we are considering FEL oscillators in which the mode coupling (active mode locking) is induced by the pulsed structure of the bunch itself. The effect was predicted long ago in Ref. [10] and is not dissimilar to the occurrence of the spikes in high gain SASE devices [11]. In this last case we
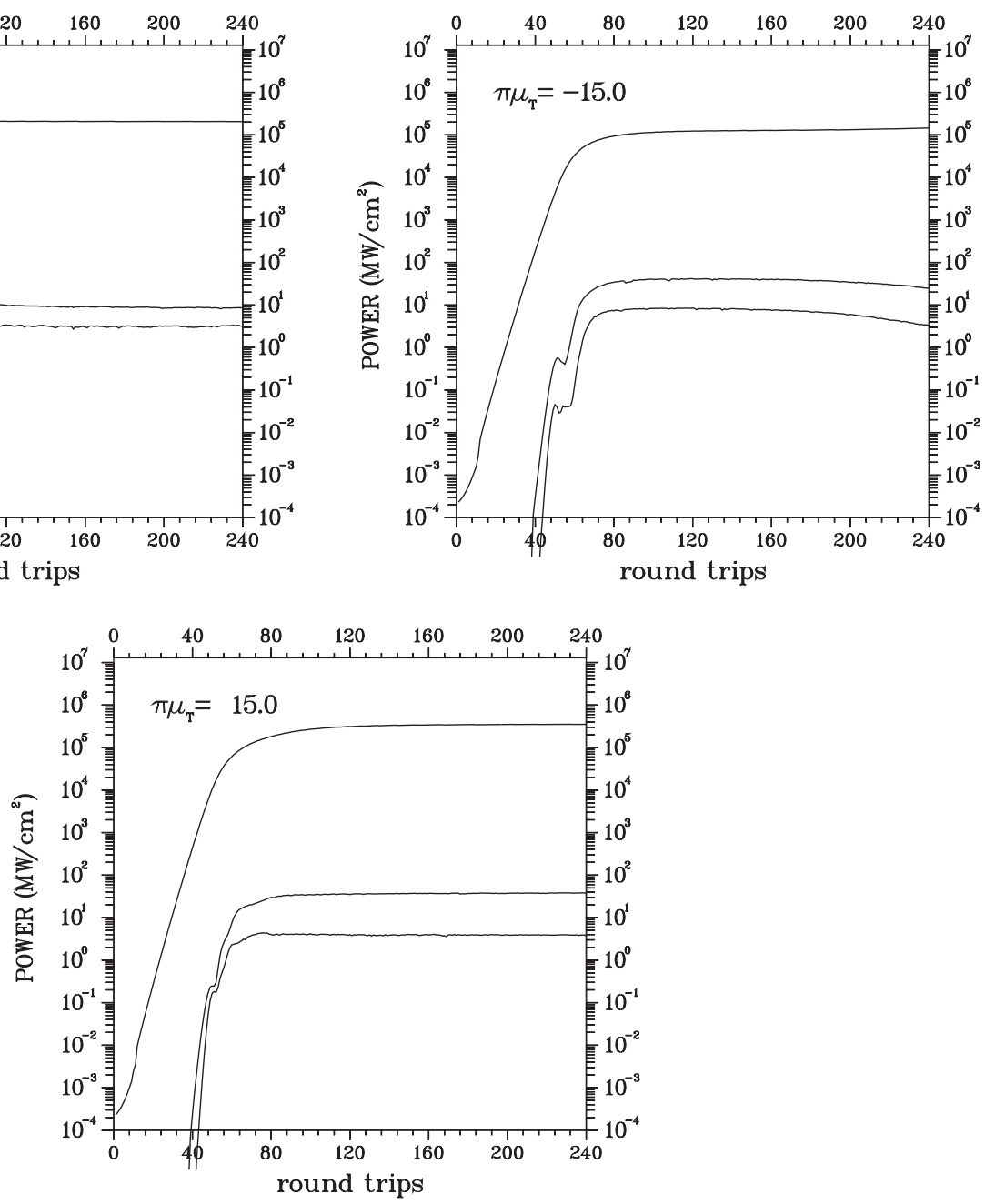

FIG. 18. Power evolution of the fundamental, third, and fifth harmonic at zero cavity mismatch for different values of $\pi \mu_{T}$. 


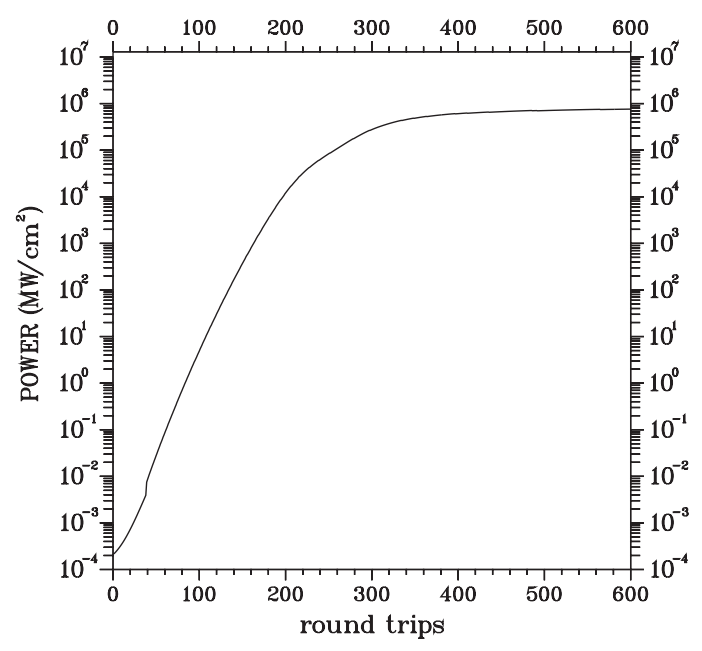

FIG. 19. Intracavity power growth at zero cavity mismatch, $g_{0}=1, \pi \mu_{T}=25, \eta=4 \%$.

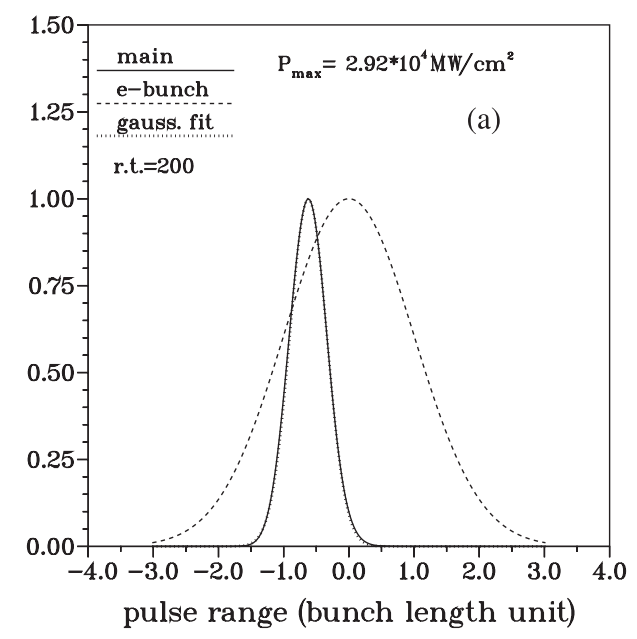

cannot however consider a mode locking in strict sense, in the absence of an optical cavity (for further comments see Ref. [12]).

This effect is also evident for the pulses associated with the higher order nonlinear harmonics, which, in the tapered case, has a less defined structure due to the fact that the optical pulse well overlapped to the electron packet is creating a strong bunching in a region spread over all the electron bunch.

The consequence of this type of structure on the coherently generated harmonics is shown in Fig. 16 where we have reported the third harmonics generated with no tapering and inverse tapering; the figures put in evidence the sensitivity of the harmonic pulse shape to the tapering, which becomes a tool to shape the emerging pulse.

A further key point is the role of the lethargy, which we have just touched on in the paper. Plots reported in Fig. 17

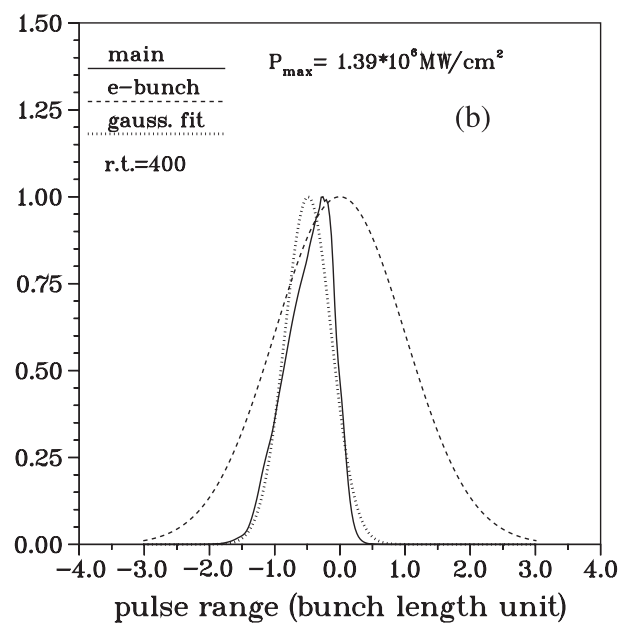

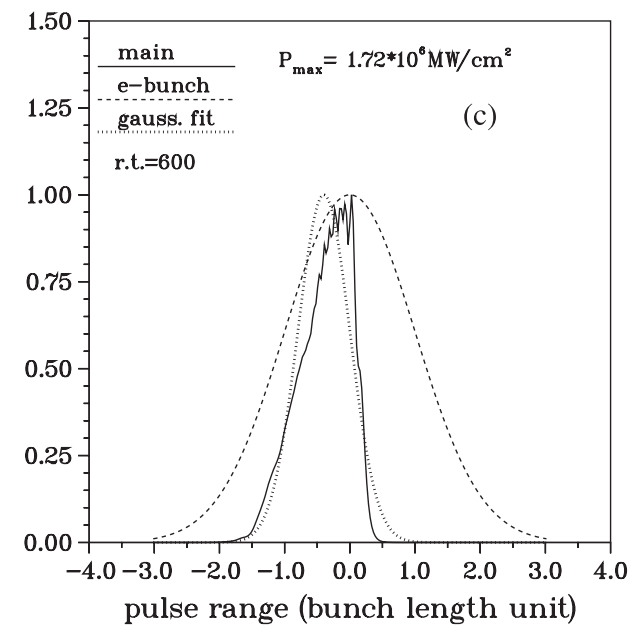

FIG. 20. Optical pulse shapes at different round-trips for $g_{0}=1, \pi \mu_{T}=25, \eta=4 \%$ : (a) round-trip 200, (b) round-trip 400, (c) round-trip 600 . 
can be considered clarifying. The figure shows indeed the behavior of the stable intracavity power vs the cavity length mismatch $\delta=\frac{\Delta L}{\lambda}$, where $\Delta L$ is the cavity length reduction necessary to compensate the lethargy effect and $\lambda$ the operating wavelength. It is evident that the reverse tapering yields the best conditions of operations, in terms of power but not for the stability curve. The region of cavity mismatch allowing the growth of stable output power is larger in the case of zero tapering. ${ }^{4}$ This is just a consequence of the fact that the gain curve is narrower in the case of reverse tapering. The case of $\pi \mu_{T}=-15$ is evidently the less favorable.

Regarding the harmonics we have reported in Fig. 18, the round-trip power evolution of the first, third, and fifth harmonic for $\pi \mu_{T}=0, \pm 15$. The effect of the tapering on the higher harmonics is quite remarkable, if confronted to the case with no tapering. In this last case the harmonic power exhibits a bump before that the fundamental has reached the onset of the saturation and then decreases even by an order of magnitude. When tapering is active, the power of the higher order harmonics remains almost constant, even when the fundamental has deeply saturated. The case with inverse tapering yields a more efficient result in terms of harmonic power too.

The final point we will treat is relevant to the case of the pulse dynamics for values of the tapering parameter giving rise to the gain instability reported in the previous sections. We have already remarked that in this region there are two competing modes which may give rise to a quite interesting dynamics and indeed we may have two different carrying frequencies separated by $\delta=\frac{\omega_{2}-\omega_{1}}{\omega_{\text {res }}} \simeq \frac{1}{N}$.

The full dynamics is rather difficult to check because the code becomes time consuming since a large number of competing modes should be included in the calculation and perhaps the slowly varying amplitude approximation is not valid any more. We have however considered the mode dynamics of a single carrying frequency with slightly larger gain with respect to the other. For such large values of the tapering, the system reaches the saturation after a large number of round-trips, as it should be, since the gain is quite low, even for large values of the small signal gain parameter (see Fig. 19).

We have noted that, at zero detuning, the slippage effect is no more active and that the optical field remains locked to the electron bunch in an almost congealed position over the entire evolution which in our simulation went well beyond the onset of the saturation (see Fig. 20). Beyond the saturation point the optical pulse becomes distorted and small structures over the peak of the bunch start to develop.

The results of this paper can be summarized as it follows: (i) FEL oscillators operating with undulators having a uniform tapering exhibit an interesting behavior associ-

\footnotetext{
${ }^{4}$ In a forthcoming investigation we will quantify more precisely these effects.
}

ated with the peculiar nature of the gain function. (ii) The uniform tapering guarantees an enhancement of the efficiency which is not the result of an optimization criterion as it happens in the case of the amplifier. (iii) The pulse propagation dynamics displays a very interesting phenomenology, which indicates that an interesting interplay may occur between tapering, slippage, and lethargy. We have some indication that the combined use of these effects can be useful to model the pulse shape.

In a forthcoming investigation, we will explore more deeply the pulse propagation dynamics in tapered oscillators and show that definite advantages (in terms of efficiency, pulse shapes, ...) can be obtained with undulators with nonuniform tapering.

\section{ACKNOWLEDGMENTS}

The authors express their sincere recognition to Dr. S. Benson for clarifying discussions on the tapered FEL and to Dr. Luca Giannessi for confirming the existence of the pulse comblike structure in deeply saturated FEL oscillators with the code PERSEO.

[1] N. Kroll, P. Morton, and M. N. Rosenbluth, IEEE J. Quantum Electron. 17, 1436 (1981).

[2] E. L. Saldin, E. A. Schneidmiller, and M. V. Yurkov, Opt. Commun. 103, 297 (1993).

[3] S. Benson, G. Biallas, C. Bohn, D. Douglas, H. F. Dylla, R. Evans, J. Fugitt, R. Hill, K. Jordan, G. Krafft, R. Legg, R. Li, L. Merminga, G. R. Neil, D. Oepts, P. Piot, J. Preble, M. Shinn, T. Siggins, R. Walker, and B. Yunn, Nucl. Instrum. Methods Phys. Res., Sect. A 429, 27 (1999).

[4] V. Asgekar, U. Lehnert, and P. Michel, 32 Free Electron Laser Conference Malmo, Sweden, 2010.

[5] G. Dattoli, M. Galli, and P. L. Ottaviani, ENEA Report No. RT/INN/93/09; G. Dattoli, S. Pagnutti, and P. L. Ottaviani "Booklet for FEL design: a collection of practical formulae" Enea Edizioni Scientifiche 2008.

[6] G. Dattoli, A. Renieri, and A. Torre, Lectures on Free Electron Laser Theory and on Related Topics (World Scientific, Singapore, 1990).

[7] W. B. Colson, in Laser Handbook, edited by W. B. Colson, C. Pellegrini, and A. Renieri (North-Holland, Amsterdam 1990), Vol. 6, Chap. 5.

[8] G. Dattoli and P. L. Ottaviani, Opt. Commun. 204, 283 (2002).

[9] A. Christodoulou, D. Lampiris, K. Polykandriotis, W. B. Colson, P. P. Crooker, S. Benson, J. Gubeli, and G. R. Neil, Phys. Rev. E 66, 056502 (2002); S. Khodyachykh, Ph.D. dissertation, University of Darmstadt, 2002.

[10] G. Dattoli and A. Renieri, Nuovo Cimento B 59, 1 (1980).

[11] E. L. Saldin, E. A. Schneidmiller, and M. V. Yurkov, The Physics of Free Electron Lasers (Springer-Verlag, Berlin, 2000).

[12] G. Dattoli, M. Delfranco, M. Labat, P. L. Ottaviani, and S. Pagnutti, http://arxiv.org/abs/1010.1647v1 (unpublished). 